Servicio de Urología Hospital DIPRECA, Santiago de Chile. ${ }^{2}$ Servicio de Urología Hospital Clínico Universidad de Chile, Santiago de Chile.

${ }^{3}$ Servicio de Urología, Instituto Nacional del Cáncer, Santiago de Chile.

aFacultad de Medicina, Universidad Diego Portales.

Declaraciones: - No se adjudicaron becas o soporte académico a este estudio.

- Declaramos la no existencia de conflictos de intereses asociados a este estudio.

- Este manuscrito es original y no ha sido publicado, ni se encuentra en revisión en ninguna revista salvo la presente.

Correspondencia a:

Camilo Novoa Brunet Vital Apoquindo 1200, Las Condes. Santiago de Chile, código postal: 7650496 camilonovoabrunet@gmail.com

\section{Nivel educacional como determinante en tamizaje de cáncer de próstata}

\author{
CAMILO NOVOA ${ }^{1}$, CAROLINA ANGUITA $^{a}$, SEBASTIÁN BADILLA-O. ${ }^{1}$, \\ ALFREDO ALIAGA ${ }^{2}$, DIEGO REYES ${ }^{2,3}$
}

\section{Prostate cancer screening is associated with educational level and knowledge about the disease}

Background: The screening of prostate cancer allows an earlier diagnosis, allowing more therapeutic options. This screening depends in part on spontaneous patients' consultation, which is largely related to their educational level. Aim: To evaluate the association between educational level, knowledge of the disease, and prostatic screening. Material and methods: A questionnaire was applied to 377 men aged between 50 and 90 years to determine their educational level, knowledge of the disease, if they had any prostate screening and age at first screening. Data was analyzed with $R$ Commander. Results: Eighty one percent of respondents had some knowledge of the disease and of these, $68 \%$ had prostate screenings compared with $34 \%$ of those without knowledge of the disease. Information about prostate cancer was reported by $71 \%$ and $96 \%$ of respondents with primary and university education, respectively. Fifty nine and $90 \%$ of respondents with primary and university education had prostate screenings performed, respectively. Conclusions: Those respondents with a prostate cancer screening had a better knowledge of the disease and a higher educational level.

(Rev Med Chile 2014; 142: 1136-1141)

Key words: Educational Status; Mass screening; Prostatic neoplasms.
E l cáncer de próstata es el segundo cáncer más diagnosticado en hombres a nivel mundial después del cáncer de pulmón, y la quinta causa de mortalidad por cáncer en hombres. A nivel mundial, el año 2012 se diagnosticaron 1,1 millones de nuevos casos de cáncer de próstata y 307.000 muertes fueron secundarias a esta causa específica ${ }^{1}$.

En Chile, el cáncer de próstata ocupa la segunda causa de muerte por cáncer en hombres, con 1.942 defunciones por esta causa específica el año 2011 y una tasa de mortalidad de 22,7 por 100.000 hombres para el mismo año ${ }^{2}$, valores que han ido en aumento sostenido durante la última década.

Debido a la elevada incidencia y mortalidad, el cáncer de próstata se encuentra incluido dentro del grupo de patologías con Garantías Explícitas en Salud (GES), que buscan asegurar una atención oportuna y de calidad en enfermedades de alta prevalencia. Sin embargo, estas garantías no incluyen el tamizaje, y sólo contemplan el tratamiento de la enfermedad una vez diagnosticada ${ }^{3}$.

Dado a esta ausencia de garantías en el tamizaje, es que la realización de éste no sólo recae en los hombros de los médicos, sino también en la de los mismos pacientes que tienen la labor de consultar. Sin embargo, el screening cuando no es de política pública y ampliamente difundido como es el caso, depende en gran medida de otros factores, y dentro de ellos el nivel educacional cumple un rol trascendental.

En estudios norteamericanos se ha visto el 
nivel educacional está fuertemente asociado con el conocimiento global acerca del cáncer de próstata $^{4}$, mientras que un nivel socioeconómico más alto, incluyendo la escolaridad entre sus factores, se asocian a una mayor información acerca del screening con antígeno prostático específico (PSA) y un inicio de controles prostáticos más temprano que pacientes con menor educación y sin seguro de salud ${ }^{5}$.

Si bien esta información podría ser extrapolada a Chile, en nuestro país no existen datos concretos respecto al grado de influencia que posee el nivel educacional respecto al conocimiento de la patología y el inicio de los controles prostáticos. Dado a esta ausencia de información, nuestro objetivo fue evaluar la asociación entre nivel educacional con respecto al conocimiento de la patología, la presencia de control prostático y su edad de inicio.

\section{Materiales y Métodos}

Estudio de tipo descriptivo, transversal. Se reclutaron sujetos de sexo masculino, con edad igual o superior a 50 años, los cuales consultaron entre junio y noviembre de 2011 por cualquier motivo a uno de los siguientes 5 centros de salud: Centro Comunitario de Salud Familiar (CECOSF) Coñimo, comuna San Joaquín; Centro de Salud Familiar (CESFAM) Sor Teresa, comuna San Joaquín; CESFAM Vitasalud, comuna Vitacura; CESFAM La Feria, comuna Pedro Aguirre Cerda; y pacientes hospitalizados del Hospital Clínico Universidad de Chile (HCUCH), comuna Independencia. Se excluyó a todos aquellos pacientes que consultaron por síntomas o patologías urológicas.

A los sujetos incluidos se les aplicó una encuesta diseñada de acuerdo a parámetros de simple entendimiento.

Las preguntas del cuestionario fueron: 1) Edad; 2) Nivel educacional completado: básica-mediatécnica-universitaria; 3) ¿Alguna vez ha leído o le han explicado sobre el cáncer de próstata y los controles prostáticos?: Sí-No; 4) ¿Se ha realizado control prostático alguna vez en su vida? Sí-No; 5) ¿Qué edad tenía la primera vez que se lo realizó?

La encuesta fue aplicada por personal entrenado a sujetos que pudieran entender la naturaleza de las preguntas y fue respondida de forma anónima por los mismos pacientes. El sistema de respuesta opcional se eligió por ser de sencillo entendimiento y rápido. Previo a la encuesta se explicó el motivo de ésta y se efectuó toma de consentimiento informado escrito visado por comité de ética de la Universidad de Chile. Al finalizar la encuesta se entregó material informativo, se dio tiempo para responder preguntas y se fomentó el control.

Los datos obtenidos fueron analizados utilizando el software R Commander y las pruebas $\mathrm{T}$ test y ANOVA, considerando estadísticamente significativo $\mathrm{p}<0,05$.

\section{Resultados}

Se reclutaron 377 sujetos que respondieron de manera voluntaria las preguntas de la encuesta. Del total de sujetos, 30,5\% tenía educación básica, $40,3 \%$ educación media, $16,2 \%$ técnica superior y $13 \%$ universitaria (Figura 1). La edad promedio de los encuestados fue 64,6 años (rango entre 50 y 90 años), sin observar diferencias estadísticas entre los promedios de edad por nivel educacional $(\mathrm{p}<0,05)$.

Respecto a la cobertura del screening, en el grupo total de encuestados se observó que 61,8\% $(\mathrm{n}=233)$ había ya iniciado algún tipo de control prostático, sin observar diferencias estadísticamente significativas en el promedio de edad entre el grupo que había iniciado controles versus los que no habían iniciado (63 versus 65,9 años, $\mathrm{p}>0,05)$.

$\mathrm{Al}$ analizar la presencia o ausencia de control prostático y la edad promedio de inicio de éste, evaluado en base a grupos educacionales, vemos

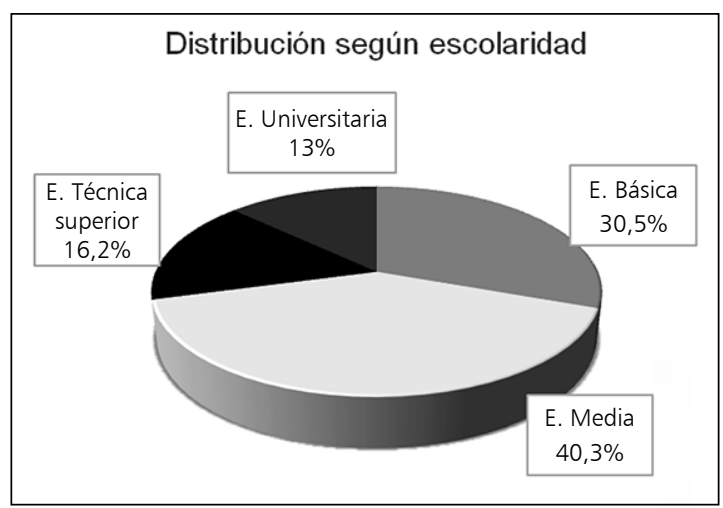

Figura 1. El gráfico muestra la distribución observada según los distintos niveles educacionales en el total de pacientes. 

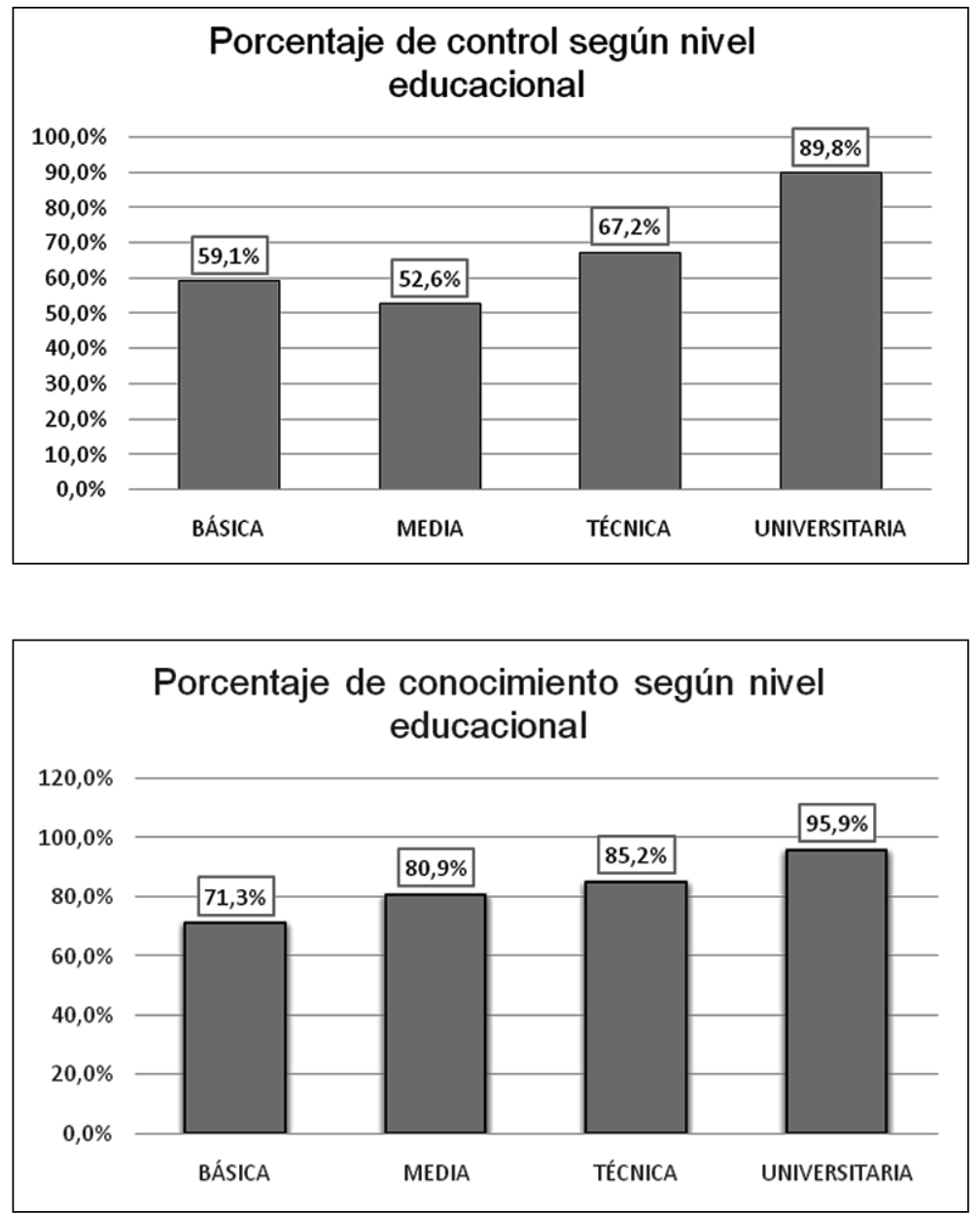

Figura 2. El gráfico de barras muestra la diferencia en la realización de screening en relación al nivel educacional de los sujetos.
Figura 3. El gráfico muestra un aumento de porcentaje de pacientes que declara conocimientos en directa relación con el nivel educacional. que: El grupo de educación básica alcanzó sólo $59,1 \%$ de cobertura de tamizaje con una edad promedio de inicio de 72,8 años. El grupo de educación media tuvo $52,6 \%$ de tamizaje con edad de inicio promedio de 54 años. El grupo técnico alcanzo $67,2 \%$ y edad promedio de 56,3 . Finalmente, el grupo universitario tuvo una cobertura de $89,8 \%$ con edad promedio de inicio a los 55 años (Figura 2). Estadísticamente hay una diferencia muy significativa al comparar el porcentaje de control de los grupos de educación básica-media versus técnica-universitaria con un $\mathrm{p}<0,001$. Respecto a la edad de inicio controles, existió diferencia estadística $\mathrm{p}<0,05$, entre el grupo de educación básico respecto a los otros niveles educacionales, pero no entre los otros grupos.

En relación al conocimiento sobre cáncer o screening prostático, cuando analizamos si los pacientes habían leído o escuchado sobre la patología también observamos diferencias: $71,3 \%$ con educación básica declaró que si habían escuchado o leído, $80,9 \%$ en educación media, $85,2 \%$ en educación técnica y $95,9 \%$ en educación universitaria (Figura 3). Estas diferencias son significativas con un $\mathrm{p}<0,05$.

Del total de pacientes, $80,6 \%$ declaró que había escuchado o leído sobre la patología, y de este subgrupo $68,4 \%$ había iniciado controles, a diferencia de $34,2 \%$ que había iniciado controles en el grupo que negó conocimientos $\mathrm{p}<0,001$ (Figura 4).

\section{Discusión}

El cáncer de próstata es un problema de salud pública mundial, situándose actualmente como la segunda causa de muerte por cáncer en hombres 


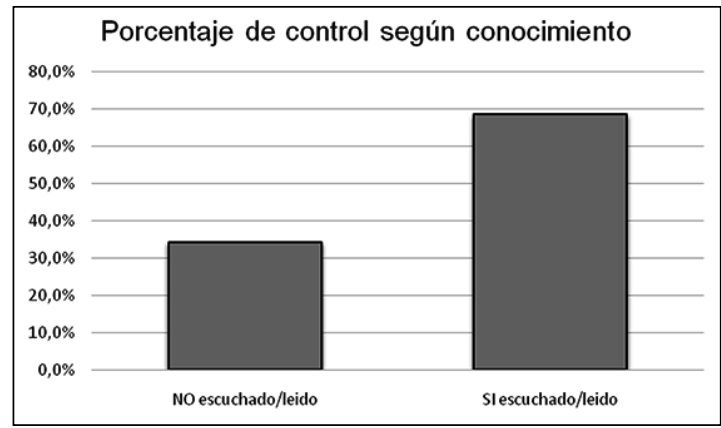

Figura 4. El gráfico muestra el porcentaje de control según conocimiento de la patología y screening.

a nivel mundial y la quinta en Chile ${ }^{1}$. Dado a que esta enfermedad por lo general no da síntomas en estadios tempranos, la pesquisa en pacientes asintomáticos es fundamental para la eficacia de terapias curativas ${ }^{6}$. Aunque ha sido puesto en duda por publicaciones recientes ${ }^{7}$, la evidencia muestra que el tamizaje para diagnóstico precoz de cáncer de próstata, utilizando el antígeno prostático específico (PSA) y el examen digital rectal, disminuye el número de tumores avanzados y la mortalidad específica por esta enfermedad ${ }^{8,9}$, y si se compara aquellos diagnosticados con screening versus clínicamente, vemos que aquellos detectados por tamizaje tienden a ser diagnosticados en etapas más tempranas y con una menor proporción de tipos histológicos pobremente diferenciados que los que se detectan clínicamente, lo que a su vez se ha asociado con tratamientos más agresivos ${ }^{10}$. Estos argumentos han sido la razón por la cual las principales sociedades científicas urológicas y oncológicas internacionales recomiendan el control prostático periódico ${ }^{11-13}$.

A pesar de lo anteriormente expuesto, en Chile no existen programas ministeriales de detección precoz que contemplen a toda la población, lo cual se ve manifiesto en que actualmente la cobertura del tamizaje no supere $50 \%$ de la población masculina $^{14}$, similar a lo ocurrido en Estados Unidos de Norteamérica o Francia ${ }^{15,16}$.

Debido a esta carencia de programas de salud que abarquen a toda la población de forma satisfactoria, el rol que cumple el paciente como consultante se vuelve mucho más relevante, especialmente en aquel grupo de pacientes carentes de otras patologías que los liguen al sistema de salud. En este mismo ámbito, dentro de los determinan- tes más importantes que surgen como influyentes en un adecuado control prostático, están tanto el nivel educacional como el nivel socioeconómico, el cual en muchos casos está ligado al factor anterior.

El screening para cáncer en general es más común en personas con mejor nivel educacional, y esto se ve reflejado en una actitud más positiva frente al screening en pacientes con un nivel educacional alto versus una actitud negativa con una situación económica desventajosa ${ }^{17}$.

Existen estudios que relacionan un mejor nivel socioeconómico/nivel educacional con un mejor conocimiento sobre la patología prostática y con un inicio más precoz del screening prostático ${ }^{4,5}$, y por la contraparte, el menor nivel socioeconómico se ha visto estar relacionado con malos resultados en pacientes con cáncer de próstata, aunque no con una mayor incidencia de cáncer, y un bajo nivel educacional con mayor mortalidad y 4,8 veces más riesgo de desarrollar metástasis. También el bajo nivel educacional se ha asociado con tratamientos menos agresivos para cáncer en etapas tempranas entre hombres mayores, así como en etapas avanzadas ${ }^{18,19}$.

En Chile no existen estudios que evalúen los controles urológicos en relación al nivel educacional, y este sería el primer trabajo en revelar una situación que si bien sospechábamos, no teníamos información concreta respecto al tema.

Los resultados revelan que sólo $61,8 \%$ de los encuestados habían iniciado algún control prostático, y esta distribución tenía directa relación con el nivel educacional de los pacientes. En el grupo de menor educación sólo 59,1\% había iniciado controles, muy por inferior de lo observado en el grupo universitario que constaba de $89,8 \%$ de screening. Importante es hacer notar que en el mismo análisis, no sólo el tamizaje era menor en los grupos con menor educación, sino que además la edad inicio de estos controles era más tardía, observándose una edad promedio de inicio de 72,8 años en la educación básica versus 55 años en la universitaria.

Lo anterior podría explicarse debido a un mayor nivel de conocimiento respecto a la enfermedad y sus controles, y así lo sugiere este estudio, donde también se observan claras diferencias entre los niveles educacionales respecto a la pregunta de conocimiento. Se observó una directa relación entre la escolaridad y el porcen- 
taje de pacientes que declararon que se les había explicado o que habían leído alguna vez sobre el cáncer de próstata y su screening, con $71,3 \%$ en el grupo de educación básica, $80,9 \%$ en educación media, $85,2 \%$ educación técnica y $95,9 \%$ en educación universitaria.

En nuestro estudio observamos que aquellos pacientes que declaraban conocimiento tenían un considerable mayor porcentaje de controles versus aquellos que no habían escuchado o leído. El 34,2\% de los pacientes sin conocimiento se había controlado, mientras que aquellos que si tenían conocimiento lo había hecho en $68,4 \%$. Esta relación entre mayor control a mayor conocimiento no puede ser interpretada como tal, ya que no podemos tener la certeza si el conocimiento es previo o posterior al screening, sin embargo, nos deja en evidencia que aquellos pacientes que declararon no haber tenido conocimiento, tienen un porcentaje de screening muy por debajo de la media observada en nuestro estudio y probablemente requiere que las medidas educacionales estén dirigidas a este grupo en particular, que además se correlaciona como vimos anteriormente con un nivel educacional menor.

Es evidente que este estudio presenta algunos puntos que se pueden considerar sesgos o reparos metodológicos. Primero, está realizado en una población que consulta a centros de salud, y no en población general. Por otro lado, si bien hay una directa relación entre el nivel educacional con el nivel socioeconómico, este último no se evalúa dirigidamente y podría ser un factor a considerar en estudio futuros. Finalmente, la interpretación de la pregunta específica en relación al conocimiento de la patología puede ser interpretada de forma subjetiva entre cada paciente, sin evaluar exactamente el conocimiento real que tiene el paciente, sin embargo, se decidió preguntar de dicha forma para evaluar más que el conocimiento específico de la patología, la percepción que tenía el paciente sobre esta, factor que puede ser mucho más determinante en ciertos casos, especialmente en lo que respecta al screening y consulta espontánea.

A pesar de estas deficiencias, este estudio deja en evidencia una clara diferencia en el screening observado entre los distintos niveles educacionales para una patología que es prioritaria en la salud nacional. Dado que modificar el nivel educacional de toda una población es un trabajo que requiere gran inversión en tiempo y dinero, creemos que la clave actual radica en una adecuada difusión de la información.

Nos parece fundamental que exista una conciencia global entre los médicos y el resto de los integrantes de la salud, que motive la constante educación de los pacientes en estos aspectos, favoreciendo de esta forma una consulta más dirigida y precoz por parte del paciente, especialmente de aquellos que poseen menores recursos educacionales y que pueden estar menos ligados al sistema de salud por diferentes motivos. Programas nacionales de educación que concienticen a la población también son una opción adecuada y complementaria a la ya expuesta. Por último, el objetivo final por el cual se debiese abogar es la implementación de un programa de screening a nivel nacional, que logre garantizar el correcto acceso a éste independiente de la situación socioeconómica del paciente, $y$ con miras a disminuir la mortalidad específica por cáncer de próstata en Chile.

\section{Referencias}

1. GLOBOCAN 2012: Estimated cancer incidence, mortality and prevalence worldwide in 2012. Disponible en: http://globocan.iarc.fr/Pages/fact_sheets_cancer.aspx (Consultado el 27 de Mayo, 2014).

2. DEIS, MINSAL, Serie de Defunciones por Tumores Malignos según Sexo, Chile 1997-2011. http://www.deis. $\mathrm{cl} /$ series-y-graficos-de-mortalidad/? $\mathrm{p}=51$ (Consultado el 26 de Mayo, 2014)-7.

3. Guía clínica Cáncer de próstata en personas de 15 años y más, Ministerio de Salud, Gobierno de Chile año 2010. Disponible en http://web.minsal.cl/portal/url/item/7222 33c6b946cd08e04001011f011d5e.pdf (consultado el 26 Mayo 2014)

4. Chan E, Vernon S, O’Donnell F, Ahn C, Greisinger A, Aga D. Informed Consent for Cancer Screening With Prostate-Specific Antigen: How Well Are Men Getting the Message? American Journal of Public Health 2003; 93 (5).

5. Steele C, Miller D, Maylahn C, Uhlen R, Baker C. Knowledge, Attitudes, and Screening Practices Among Older Men Regarding Prostate Cancer. American Journal of Public Health 2000; 90 (10).

6. Roehl KA, Han M, Ramos CG, Antenor JA, Catalona WJ. Cancer progression and survival rates following anatomical radical retropubic prostatectomy in 3,478 consecutive patients: long-term results. J Urol 2004; 172 (3): 910-4.

7. Andriole GL, Crawford ED, Grubb RL 3rd, Buys SS, 
Chia D, Church TR, et al. Mortality results from a randomized prostate-cancer screeningtrial. N Engl J Med 2009; 360 (13): 1310-9.

8. Helgesen F, Holmberg L, Johansson JE, Bergström R, Adami HO. Trends in prostate cancer survival in Sweden, 1960 through 1988, evidence of increasing diagnosis of non-lethal tumours. J Natl Cancer Inst 1996; 88 (17): 1216-21.

9. Bartsch G, Horninger W, Klocker H, Reissigl A, Oberaigner W, Schönitzer D, et al. Tyrol Prostate Cancer Screening Group. Prostate cancer mortality after introduction of prostate specific antigen mass screening in the Federal State of Tyrol, Austria. Urology 2001; 58 (3): 417-24.

10. Hoffman R, Stone N, Espey D, Potosky A. Differences between men with screening-detected versus clinically diagnosed prostate cancers in the USA. BMC Cancer 2005; 5: 27.

11. Guía clínica sobre el cáncer de próstata, AEU. Disponible en: http://www.aeu.es/guiasaeueua.aspx (Consultado el 25 de Mayo 2014).

12. National Comprehensive Cancer Network Clinical Practice Guidelines in Oncology. Prostate Cancer Early Detection. Version 1. 2014. Disponible en: http://www. nccn.org/professionals/physician_gls/pdf/prostate_detection.pdf (Consultado el 26 de Mayo 2014).

13. Early Detection of Prostate Cancer: American Urologi- cal Association guidelines 2013. Disponible en:https:// www.auanet.org/education/guidelines/prostate-cancerdetection.cfm (Consultado el 26 de Marzo, 2014)

14. Novoa C, Aliaga A, Badilla S, Reyes D. Realidad actual del screening para cáncer de próstata. ¿Se llevan a cabo las recomendaciones? Revista Chilena de Urología 2013; 78 (4): 27-31.

15. Kadiyala S, Strumpf EC. Are United States and Canadian cancer screening rates consistent with guideline information regarding the age of screening initiation? Int J Qual Health Care 2011; 23 (6): 611-20.

16. Tuppin P, Samson S, Perrin P, Ruffion A, Millat B, Weill A, et al. Prostate-Specific Antigen use among men without prostate cancer in France (2008-2010). Bull Cancer 2012 Apr 23.

17. Cullati S, Charvet-Bérard A, Perneger T. Cancer screening in a middle-aged general population: factors associated with practices and attitudes. BMC Public Health 2009; 9: 118 .

18. Gilligan T. Social disparities and prostate cancer: mapping the gaps in our knowledge. Cancer Causes and Control 2005; 16: 45-53.

19. de Souza A, Guedes H, Oliveira V, Aires de Araújo F, Oliveira C, Medeiros K, et al. High incidence of prostate cancer metastasis in Afro-Brazilian men with low educational levels: a retrospective observational study. BMC Public Health 2013; 13: 537. 